\title{
Genetic Association of NPY Gene Polymorphisms with Dampness-Phlegm Pattern in Korean Stroke Patients
}

\author{
Mi Mi Ko, Byoung Kab Kang, Ji Hye Lim, Myeong Soo Lee, and Min Ho Cha \\ Brain Disease Research Center, and Division of Standard Research, Korea Institute of Oriental Medicine, 1672 Yuseongdae-ro, \\ Yuseong-gu, Daejeon 305-811, Republic of Korea \\ Correspondence should be addressed to Min Ho Cha, mhchamin@kiom.re.kr
}

Received 23 June 2011; Accepted 2 October 2011

Academic Editor: Jae Youl Cho

Copyright ( 2012 Mi Mi Ko et al. This is an open access article distributed under the Creative Commons Attribution License, which permits unrestricted use, distribution, and reproduction in any medium, provided the original work is properly cited.

Neuropeptide Y (NPY), which is widely expressed in both the central and peripheral nervous systems, has an important role in a variety of biological fields. In this study, we analyzed the distribution of NPY polymorphisms in dampness-phlegm pattern and non-dampness-phlegm pattern in elderly Korean subjects with cerebral infarction (CI). A total of 1.097 subjects (498 normal subjects and 599 CI patients, including 198 with dampness-phlegm pattern and 401 with non-dampness-phlegm pattern) participated in this study. Genotyping for five SNPs (G-1484A, C-1471T, C-399T, A1201G, and C5325T) was conducted by primer extension. The results were statistically analyzed for genetic association of NPY-polymorphisms with normal versus dampnessphlegm pattern or non-dampness-phlegm pattern subjects. Among the five SNPs tested, the T allele of C-399T has a negative association with the dampness-phlegm pattern and is marked by a decrease in serum cholesterol levels. Furthermore, serum cholesterol levels were significantly higher in dampness-phlegm pattern patients than in non-dampness-phlegm pattern patients.In this study, for the first time, the association of NPY polymorphisms with pattern identification (PI) of traditional Korean medicine (TKM) was analyzed in a large CI patient population.

\section{Introduction}

Neuropeptide Y (NPY), first identified in mammalian brains in the 1980s, is a neuropeptide consisting of 36 amino acids $[1,2]$ that exhibits diverse biological functions [3]. Most of the NPY protein is secreted by the peripheral or central nervous systems [1] and is involved in multiple physiological processes and pathological conditions, including memory, stress, overeating and obesity, pain regulation, neural regulation, Alzheimer's disease, regulation of blood pressure, and cerebral circulation [3-13]. Direct or indirect effects of NPY have been particularly implicated in obesity and metabolic syndromes. Largely secreted NPY stimulates eating and drives overeating-induced obesity [14]. Furthermore, the expression and secretion of NPY by sympathetic nerves and fat tissue was recently shown to activate proliferation and adipogenesis of preadipocytes in fat tissue [15-17].

The NPY gene, located at chromosome $7 \mathrm{p} 15.1$, has twenty-five polymorphisms in its promoter region and seven within its exons (NT_007819.17). The L7P SNP has been associated with type 2 diabetes mellitus, alcoholism, and obesity [18-22]. Another functional SNP, C-399T, in which the $\mathrm{T}$ allele exhibits lower transcriptional activity than the $\mathrm{C}$ allele, was also associated with schizophrenia [23] and nonHodgkin's lymphoma [24]. Recently, Kim et al. reported that this SNP was significantly associated with ischemic stroke in the Korean elderly population [25], with Yu et al. showing the same results in Chinese subjects [26].

Traditional Korean medicine (TKM), which is similar to traditional Chinese medicine (TCM), categorizes stroke into internal disease symptoms. Pattern identification (PI) is a diagnostic system that entails a comprehensive analysis of symptoms and signs, with implications for determining the cause, nature, and location of the illness; the patient's physical condition; the patient's treatment [27, 28]. Go previously reported that the PI of stroke in TKM can be classified into five types: fire heat pattern, dampness-phlegm pattern, blood stasis pattern, yin deficiency pattern and Qi deficiency pattern [29]. Among these, subjects with dampness-phlegm pattern tend to be obese and have hyperlipidemia [30]. 
PI is affected by environmental and hereditary factors. Some reports have suggested a relationship between genetic variation and PI among Chinese, Korean, and Japanese populations [31-34].

In this study, we analyzed the distribution of NPY polymorphisms in dampness-phlegm pattern and nondampness-phlegm pattern in elderly Korean subjects.

\section{Materials and Methods}

2.1. Study Subjects. Patients with cerebral infarction (CI) were admitted into twelve Korean oriental medical hospitals participating in this study: Kyung Hee Oriental Medical Center (Seoul), Kyung Hee East-West Neo Medical Center (Seoul), Dong Guk International Hospital (Kyunggi-do), Kyung Won Oriental Medical Hospitals (Seoul and Incheon), Dong Seo Oriental Medical Hospital (Seoul), Dae Jeon Oriental Medical Hospital (Daejeon), Dong Sin Oriental Medical Hospital (Gwangju and Jeollanam-do), Won Kwang Oriental Medical Hospital (Jeollabuk-do), Woo Suk Oriental Medical Hospital (Jeollabuk-do), and Sang Ji Oriental Medical Hospital (Gangwon-do). CI was confirmed by magnetic resonance imaging (MRI) or magnetic resonance angiography (MRA). After obtaining informed consent from all subjects, clinical data were collected, and syndromes were differentiated classified using "stroke PI case report form." PI diagnosis of each patient was determined by two expert TKM doctors, and subjects receiving differing opinions from two doctors were excluded [27]. Patients with histories of transient ischemic attack (TIA), diabetes, hyperlipidemia, and heart disease were excluded, as were patients with a previous stroke or a traumatic stroke.

Normal subjects were recruited from Dae Jeon Oriental Medical Hospital and Won Kwang Oriental Medical Hospital, and stroke status was confirmed by MRI. Subjects with a history of stroke, TIA, diabetes, hyperlipidemia and heart disease were excluded. Four hundred ninety-eight normal subjects and five hundred ninety-nine CI patients classified as having dampness-phlegm pattern $(N=198)$ or non-dampness-phlegm pattern $(N=401)$ participated in this study. The general characteristics of normal subjects and CI patients are shown in Supplemental Table 1 (See in Supplementary Material available online at doi:10.1155/2012/109796.). This study was approved by the Institutional Review Boards of the Korean institute of Oriental Medicine and by each of the Oriental Medical Hospitals.

2.2. Preparation of Genomic DNA and Identification of SNPs. Genomic DNA of each subject was extracted from whole blood using a GeneAll Genomic DNA Extraction Kit (GeneAll, Seoul, Korea). The promoter region $(2 \mathrm{~kb})$ and all exons of the NPY gene were sequenced in 24 Korean genomic DNA samples using an ABI PRISM 3700 DNA analyzer (Applied Biosystems, Foster City, Calif, USA). Primer sets used in the amplification and sequencing analyses were designed on the basis of the reference genome sequence for NPY (NC_000007). Information concerning the primers for the amplification and sequencing of the NPY gene is shown in Supplemental Table 2.

Genotyping of SNPs in all subjects was conducted using the SNaPshot multiplex system (Applied Biosystem, FosterCity, CA, USA) according to the manufacturer's protocol. Primer and NPY SNP genotyping probe sequences are shown in Supplemental Table 3.

Hardy-Weinberg equilibrium tests were employed to determine whether individual SNPs were in equilibrium at each locus, and linkage disequilibrium (LD) coefficients, $\left|D^{\prime}\right|$ and $r^{2}$, were evaluated to measure LD between all pairs of loci [35]. The frequencies of SNPs and LD coefficients were inferred using the HapAnalyzer program v1.0.

2.3. Statistical Analysis. Data were statistically analyzed with SAS software, version 9.1.3 (SAS Institute Inc., Cary, NC). All continuous variables were subjected to a KolmogorovSmirnov normality test. Differences in continuous variables were determined by parametric (Student's $t$-test) or nonparametric (Wilcoxon rank sum test) test. Categorical variables were compared with a chi-square test or Fisher's exact test.

Multiple logistic regression adjusted for age, sex, smoking status, and drinking status was performed to estimate the association of SNPs with normal versus dampness-phlegm pattern (or non-dampness-phlegm pattern), as well as odds ratios (ORs) with $95 \%$ confidence intervals (95\% CI). To investigate whether the C-399T polymorphism is associated with the clinical parameters of the normal subjects, we performed a statistical analysis using a general linear model adjusted for age, sex, smoking status, and drinking status. Statistical significance was set at $P<0.05$.

\section{Results}

General characteristics of normal and CI patients are shown in Supplemental Table 1 and are similar to our previous study [25]. The clinical differences between the CI patients classified as dampness-phlegm pattern or non-dampnessphlegm pattern according to the PI of TKM are shown in Table 1 . The mean waist circumference of the dampnessphlegm pattern patients was significantly higher than that of the normal subjects $(P<0.001)$. A higher mean waist circumference was also observed in the dampness-phlegm pattern group compared with the non-dampness-phlegm pattern group. Additionally, serum lipids, total cholesterol and LDL-cholesterol levels were higher in the dampnessphlegm pattern group than in the non-dampness-phlegm pattern group.

The location of five SNPs within the NPY gene are shown in Figure 1(a), and their characteristics is listed in Table 2. Three of these SNPs are in the NPY promoter, one is in exon 2, and the other is in exon 3. None of the alleles result in an amino acid change. All of the alleles were in Hardy-Weinberg equilibrium $(P>0.01)$ according to the recommended International HapMap Project guidelines. The LD coefficients between the five SNPs are 
TABLE 1: Demographic parameters of study subjects.

\begin{tabular}{|c|c|c|c|c|c|}
\hline $\begin{array}{l}\text { Characteristics } \\
N\end{array}$ & $\begin{array}{c}\text { Normal } \\
498\end{array}$ & $\begin{array}{l}\text { Non-dampness-phlegm } \\
401\end{array}$ & sig. $^{\text {a }}$ & $\begin{array}{c}\text { Dampness-phlegm } \\
198\end{array}$ & sig. \\
\hline \multicolumn{6}{|l|}{ Anthropometric characteristics } \\
\hline $\operatorname{Sex}(M / F)$ & $215 / 283$ & $226 / 175$ & $<.0001$ & $104 / 94$ & $<.05$ \\
\hline Age (year) & $60.76 \pm 9.77$ & $67.02 \pm 11.94$ & $<.0001$ & $64.7 \pm 12.81$ & $<.0001$ \\
\hline Smoking (none/stop/active) & $241 / 214 / 43$ & $216 / 81 / 104$ & $<.0001$ & $117 / 21 / 60$ & $<.0001$ \\
\hline Drinking (none/stop/active) & $298 / 31 / 168$ & $199 / 59 / 143$ & $<.0001$ & $114 / 14 / 69$ & NS \\
\hline WHR & $0.88 \pm 0.06$ & $0.93 \pm 0.07$ & $<.0001$ & $0.93 \pm 0.05$ & $<.0001$ \\
\hline Waist circumference $(\mathrm{cm})$ & $83.61 \pm 8.38$ & $84.61 \pm 9.79$ & NS & $87.13 \pm 9.82$ & $<.001$ \\
\hline \multicolumn{6}{|l|}{ TOAST classification } \\
\hline LAA & - & 64 & $\mathrm{ND}$ & 30 & ND \\
\hline CE & - & 15 & & 8 & \\
\hline SVO & - & 237 & & 123 & \\
\hline SOE & - & 9 & & 2 & \\
\hline SUE & 一 & 14 & & 4 & \\
\hline \multicolumn{6}{|l|}{ Medical history } \\
\hline Hypertension (Yes, \%) & $60(12.07)$ & $217(54.94)$ & $<.0001$ & $120(60.61)$ & $<.0001$ \\
\hline \multicolumn{6}{|l|}{ Blood parameter } \\
\hline GOP (U/mL) & $27.56 \pm 19.97$ & $27.31 \pm 22.5$ & NS & $26.08 \pm 16.07$ & NS \\
\hline GPT $(\mathrm{U} / \mathrm{mL})$ & $26.04 \pm 20.26$ & $25.6 \pm 24.29$ & NS & $26.19 \pm 23.37$ & NS \\
\hline Total cholesterol (mg/dL) & $202.23 \pm 40.04$ & $181.5 \pm 43.68$ & $<.0001$ & $192.83 \pm 43.95$ & $<.01$ \\
\hline Triglyceride (mg/dL) & $139.88 \pm 69.80$ & $149.86 \pm 95.72$ & NS & $150.75 \pm 101.28$ & NS \\
\hline HDL-cholesterol (mg/dL) & $52.28 \pm 12.69$ & $46.1 \pm 14.33$ & $<.0001$ & $44.57 \pm 12.44$ & $<.0001$ \\
\hline LDL-cholesterol (mg/dL) & $121.19 \pm 36.70$ & $109.42 \pm 36.75$ & $<.0001$ & $121.21 \pm 40.35$ & NS \\
\hline Bloog sugar (mg/dL) & $99.75 \pm 10.79$ & $112.05 \pm 34.48$ & $<.0001$ & $109.81 \pm 29.53$ & $<.0001$ \\
\hline
\end{tabular}

All results except sex, TOAST categories, smoking, drinking, and hypertension are expressed as mean \pm SD for continuous variables. NS: not significant. ND: not done. WHR: waist hip ratio. TOAST: trial of ORG 10172 in acute stroke treatment. LAA: large-artery atherosclerosis. CE: cardioembolism. SVO: smallvessel occlusion. SOE: stroke of other etiology. SUE: stroke of undetermined etiology. GOP: glutamate oxaloacetate transaminase. GPT: glutamate pyruvate transaminase. sig. ${ }^{a}: P$ value of normal versus non-dampness-phlegm using a Student's $t$-test or Wilcoxon rank-sum test in continuous variables, chi-square test, or Fisher's exact test in categorical variables. sig. ${ }^{b}: P$ value of normal versus dampness-phlegm using a Student's $t$-test or Wilcoxon rank sum test in continuous variables, chi-square test, or Fisher's exact test in categorical variables. $P$ values with statistical significance were presented in bold.

TABLE 2: Characteristics of SNPs identified by genomic sequencing of the promoter, exon of NPY in normal group.

\begin{tabular}{|c|c|c|c|c|c|c|c|}
\hline SNP & rs no. & Position & $\begin{array}{l}\text { Location relative to } \\
\text { transcription start site }\end{array}$ & $\begin{array}{c}\text { Nucleotide } \\
\text { change }\end{array}$ & $\begin{array}{l}\text { Location relative to } p \\
\text { terminus of chromosome }\end{array}$ & $\begin{array}{l}\text { Minor allele } \\
\text { frequency }\end{array}$ & HWE $p$ \\
\hline G-1484A & rs16149 & Promoter & -2535 & $\mathrm{G}>\mathrm{A}$ & $24,322,325$ & 0.314 & 0.2624 \\
\hline C-1471T & rs16148 & Promoter & -2522 & $\mathrm{C}>\mathrm{T}$ & $24,322,338$ & 0.171 & 0.2733 \\
\hline C-399T & rs16147 & Promoter & -1450 & $\mathrm{C}>\mathrm{T}$ & $24,323,410$ & 0.331 & 0.0709 \\
\hline A1201G & rs5573 & Exon 2 & 150 & $A>G$ & $24,325,009$ & 0.342 & 0.0417 \\
\hline C5325T & rs5574 & Exon 3 & 4274 & $\mathrm{C}>\mathrm{T}$ & $24,329,133$ & 0.314 & 0.1836 \\
\hline
\end{tabular}

shown in Figure 1(b). Among these, G-1484A, C-399T, and A1201G were slightly linked $\left(\left|D^{\prime}\right|=1\right.$ and $r^{2}=$ $0.862-0.951)$.

Table 3 shows the SNP distribution in the nondampness-phlegm pattern and the dampness-phlegm pattern groups compared with the normal group. The frequency of T allele of C-399T is $27.27 \%$ in dampness-phlegm pattern, which is significantly lower than in normal (33\%) and nondampness-phlegm pattern patients (45.96\%), as adjusted for sex, age, smoking, drinking and hypertension $[P=0.0378$, $\mathrm{OR}=0.737(0.552-0.983)]$. The ratio of subjects with $\mathrm{T}$ allele in the dampness-phlegm pattern group $(45.96 \%)$ is also smaller than in the normal (53.25\%) and nondampness-phlegm pattern groups $(56.0 \%)[P=0.0315$, $\mathrm{OR}=0.663(0.456-0.964)]$. In contrast, subjects with $\mathrm{T}$ allele of $\mathrm{C} 5325 \mathrm{~T}$ at exon 3 exhibited significantly more dampness-phlegm pattern than normal and non-dampnessphlegm pattern in the dominant model.

Table 4 shows the comparison of obesity phenotypes and serum lipids in C-399T genotypes. The level of total cholesterol and LDL-cholesterol in normal subjects with TT type at C-399T was significantly lower than subjects with $\mathrm{C}$ allele $(P=0.0111$ and $P=0.0186$, resp. $)$. Conversely, triglycerides were slightly increased in the dominant model. 


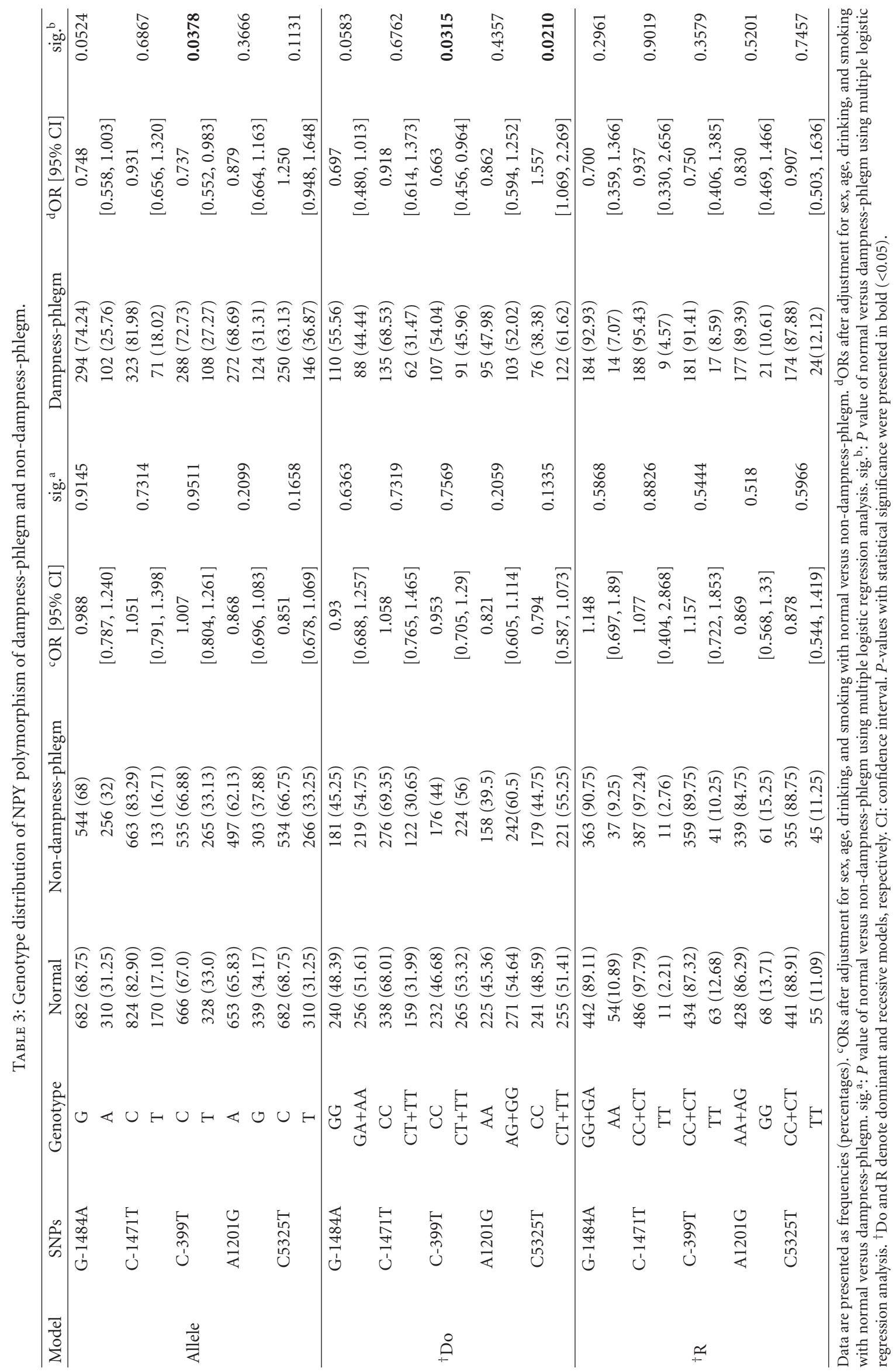




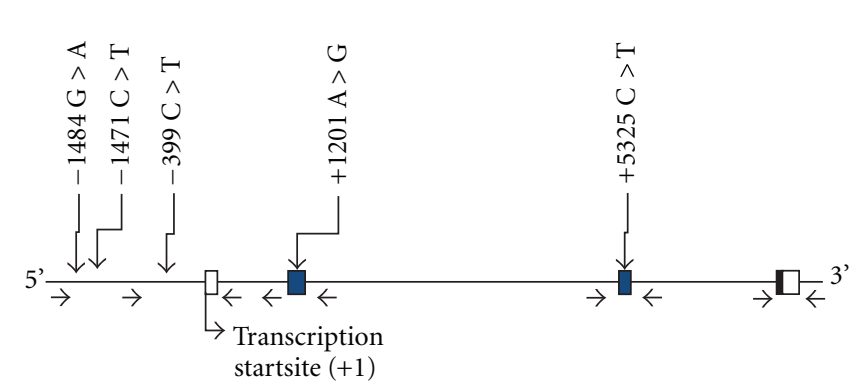

(a)

\begin{tabular}{rr|ccccc} 
& & \multicolumn{5}{|c}{$\left|D^{\prime}\right|$} \\
& & G-1484A & C-1471T & C-399T & A1201G & C5325T \\
\hline G-1484A & & 1.000 & 0.980 & 0.90 & 1.000 \\
& C-1471T & 0.094 & & 0.904 & 0.875 & 0.949 \\
$r^{2}$ & C-399T & 0.888 & 0.083 & & 1.000 & 1.000 \\
& A1201G & 0.862 & 0.082 & 0.951 & & 0.973 \\
& C5325T & 0.209 & 0.407 & 0.226 & 0.225 & \\
\hline
\end{tabular}

(b)

FIgURE 1: Polymorphism in NPY. (a) Location of SNPs. Open box and closed box represent non translated region and translated region, respectively. Primers for genomic amplification and sequencing are shown below the gene map. (b) Linkage coefficiency among five SNPs of NPY.

TABLE 4: Association of NPY polymorphism (C-399T) with clinical parameters of normal subjects.

\begin{tabular}{|c|c|c|c|c|c|c|}
\hline \multirow{2}{*}{ Variable } & \multicolumn{3}{|c|}{ Genotype } & \multicolumn{3}{|c|}{$P$} \\
\hline & $\mathrm{CC}(n=232)$ & $\mathrm{CT}(n=202)$ & $\mathrm{TT}(n=63)$ & ${ }^{\dagger} \mathrm{Co}$ & ${ }^{\dagger}$ Do & ${ }^{\dagger} \mathrm{R}$ \\
\hline Waist & $82.8 \pm 8.43$ & $84.46 \pm 8.5$ & $83.58 \pm 7.47$ & 0.4988 & 0.3007 & 0.8857 \\
\hline WHR & $0.88 \pm 0.07$ & $0.88 \pm 0.05$ & $0.87 \pm 0.05$ & 0.4535 & 0.3418 & 0.2656 \\
\hline Total cholesterol & $199.27 \pm 37.97$ & $209.11 \pm 42.71$ & $190.79 \pm 35.25$ & 0.0019 & 0.1748 & 0.0111 \\
\hline Triglyceride & $132.15 \pm 66.49$ & $147.14 \pm 74.71$ & $143.49 \pm 63.13$ & 0.0869 & 0.03 & 0.697 \\
\hline HDL-cholesterol & $52.9 \pm 13.05$ & $52.79 \pm 12.55$ & $50.89 \pm 11.86$ & 0.3289 & 0.3076 & 0.1666 \\
\hline LDL-cholesterol & $119.32 \pm 35.21$ & $126.38 \pm 39.09$ & $111.2 \pm 31.91$ & 0.0076 & 0.2913 & 0.0186 \\
\hline Blood sugar & $99.33 \pm 9.77$ & $99.95 \pm 11.63$ & $100.7 \pm 11.76$ & 0.4249 & 0.1984 & 0.4885 \\
\hline
\end{tabular}

Data are presented as mean \pm S.D. $P$ values adjusted odds ratio, adjustment for sex, age, drinking, and smoking using a general linear model. Statistical significance was presented in bold $(<0.05) .{ }^{\dagger} \mathrm{Co}$, Do, and R denote codominant, dominant, and recessive models, respectively.

\section{Discussion}

PI is the basic system for diagnosis of patients in TKM, and it entails a systematic analysis of the patient's physical condition with implications for determination of the cause, nature, location, and treatment of the illness. The PI can lead TKM doctors to provide their patients with individualized treatment, thereby increasing the effectiveness of TKM and minimizing its adverse effects.

In Korea, several questionnaires for the standardization of PI have been developed, the recent establishment of five standard PI subtypes for stroke: fire heat pattern, dampnessphlegm pattern, blood stasis pattern, Yin deficiency pattern and Qi deficiency pattern [29, 36]. Among these, the dampness-phlegm pattern is characterized by its impediment to Qi movement and its turbidity, heaviness, stickiness, and downward-flowing properties [17]. Moreover, Kim et al. [27] reported that pale tongue, slippery pulse, and overweight status are major factors positively associated with dampnessphlegm pattern. Other studies showed that Korean stroke patients with dampness-phlegm pattern had increased serum cholesterol levels [30]. We also obtained similar results in this study (Table 1). Collectively, these factors suggest that genes or genetic variation related with obesity might be associated with dampness-phlegm pattern in stroke.

In the current study, we examined the association of the dampness-phlegm pattern of stroke with five SNPs of the
NPY gene among Korean CI patients and found that the C399T SNP was negatively associated with dampness-phlegm pattern $(\mathrm{OR}=0.663)$ (Table 3$)$.

NPY, which is widely expressed in both the central and peripheral nervous systems, has an important role in the hypothalamic regulation of energy balance by stimulating food intake and favoring energy storage through increased lipoprotein lipase activity in white adipose tissue [14].

Many groups have studied the association of NPY polymorphisms with obesity and/or serum lipid levels. Karvonen et al. [37] reported that the presence of the $\mathrm{P}$ allele of L7P SNP was associated with higher serum levels of total and LDL-cholesterol in obese and normal-weight subjects, and others have shown that L7P SNP is associated with increased body mass index (BMI) [38]. However, this polymorphism has not been found in Asian populations $[39,40]$.

Plasma NPY level is positively correlated with total cholesterol and LDL-cholesterol levels [41], and our results show that the C-399T SNP is significantly associated with decreased serum total cholesterol and LDL-cholesterol in normal subjects (Table 4). Recently, four studies found that the C-399T SNP affects NPY level. We previously reported that the T allele of C-399T decreases NPY transcriptional activity and that the plasma NPY level of subjects with the TT type was significantly decreased compared with subjects with the CC or CT type [25]. In addition, three other 
groups documented the allele-specific effects of the C-399T polymorphism on NPY gene expression [42, 43]. Taken together, these data suggest that subjects with the TT type of C-399T may have lower levels of serum total cholesterol and LDL-cholesterol, due to decreased plasma NPY levels.

In this study, for the first time, the association of NPY polymorphisms with PI of TKM was analyzed in a large CI patient population. Among the five SNPs, the $\mathrm{T}$ allele of $\mathrm{C}$-399T has a negative association with dampness-phlegm pattern by decreased levels of serum cholesterol, which were significantly higher in dampness-phlegm pattern patients compared with non-dampness-phlegm pattern patients. It is clear that the findings of this study should be interpreted within the context of its limitations. Our findings are not appropriate for extrapolation to the general population. The observed associations, therefore, will require further confirmation in other subject groups.

\section{Acknowledgment}

This research was supported by a Grant from the Korea Institute of Oriental Medicine (K11132).

\section{References}

[1] S. H. Chen, P. C. W. Fung, and R. T. F. Cheung, "Neuropeptide Y-Y1 receptor modulates nitric oxide level during stroke in the rat," Free Radical Biology and Medicine, vol. 32, no. 8, pp. 776784, 2002.

[2] S. M. Wallerstedt, S. Skrtic, A. -L. Eriksson, C. Ohlsson, and T. Hedner, "Association analysis of the polymorphism T1128C in the signal peptide of neuropeptide $\mathrm{Y}$ in a Swedish hypertensive population," Journal of Hypertension, vol. 22, no. 7, pp. 12771281, 2004.

[3] J. G. Wettstein, B. Earley, and J. L. Junien, "Central nervous system pharmacology of neuropeptide Y," Pharmacology and Therapeutics, vol. 65, no. 3, pp. 397-414, 1995.

[4] J. E. Morley and J. F. Flood, "Neuropeptide Y and memory processing," Annals of the New York Academy of Sciences, vol. 611, pp. 226-231, 1990.

[5] M. Heilig, G. F. Koob, R. Ekman, and K. T. Britton, "Corticotropin-releasing factor and neuropeptide Y: role in emotional integration," Trends in Neurosciences, vol. 17, no. 2, pp. 80-85, 1994.

[6] M. Heilig, S. McLeod, M. Brot et al., "Anxiolytic-like action of neuropeptide Y: mediation by Y1 receptors in amygdala, and dissociation from food intake effects," Neuropsychopharmacology, vol. 8, no. 4, pp. 357-363, 1993.

[7] X. Y. Hua, J. H. Boublik, M. A. Spicer, J. E. Rivier, M. R. Brown, and T. L. Yaksh, "The antinociceptive effects of spinally administered neuropeptide $\mathrm{Y}$ in the rat: systematic studies on structure-activity relationship," Journal of Pharmacology and Experimental Therapeutics, vol. 258, no. 1, pp. 243-248, 1991.

[8] J. F. Flood and J. E. Morley, "Dissociation of the effects of neuropeptide $\mathrm{Y}$ on feeding and memory: evidence for pre- and postsynaptic mediation," Peptides, vol. 10, no. 5, pp. 963-966, 1989.

[9] R. J. Beninger, B. A. Wirsching, K. Jhamandas, and R. J. Boegman, "Animal studies of brain acetylcholine and memory," Archives of Gerontology and Geriatrics, vol. 8, no. 1, pp. 71-89, 1989.
[10] U. I. Tuor, P. A. T. Kelly, L. Edvinsson, and J. McCulloch, "Neuropeptide Y and the cerebral circulation," Journal of Cerebral Blood Flow and Metabolism, vol. 10, no. 5, pp. 591601, 1990.

[11] P. S. Widdowson and A. E. Halaris, "Increased levels of neuropeptide Y-immunoreactivity in rat brain limbic structures following antidepressant treatment," Journal of Neurochemistry, vol. 52, p. S77, 1989.

[12] S. O. Frederiksen, R. Ekman, C. G. Gottfries, E. Widerlov, and $\mathrm{S}$. Jonsson, "Reduced concentrations of galanin, arginine vasopressin, neuropeptide $Y$ and peptide YY in the temporal cortex but not in the hypothalamus of brains from schizophrenics," Acta Psychiatrica Scandinavica, vol. 83, no. 4, pp. 273-277, 1991.

[13] S. M. Stahl, Essential Psychopharmacology: Neuroscientific Basis and Clinical Applications, Cambridge University Press, Cambridge, UK, 2nd edition, 2000.

[14] B. Beck, "Neuropeptide Y in normal eating and in genetic and dietary-induced obesity," Philosophical Transactions of the Royal Society B: Biological Sciences, vol. 361, no. 1471, pp. 1159-1185, 2006.

[15] L. E. Kuo, J. B. Kitlinska, J. U. Tilan et al., "Neuropeptide Y acts directly in the periphery on fat tissue and mediates stressinduced obesity and metabolic syndrome," Nature Medicine, vol. 13, no. 7, pp. 803-811, 2007.

[16] K. Kos, A. L. Harte, S. James et al., "Secretion of neuropeptide $\mathrm{Y}$ in human adipose tissue and its role in maintenance of adipose tissue mass," American Journal of PhysiologyEndocrinology and Metabolism, vol. 293, no. 5, pp. E1335E1340, 2007.

[17] K. Yang, H. Guan, E. Arany, D. J. Hill, and X. Cao, "Neuropeptide $\mathrm{Y}$ is produced in visceral adipose tissue and promotes proliferation of adipocyte precursor cells via the Y1 receptor," FASEB Journal, vol. 22, no. 7, pp. 2452-2464, 2008.

[18] J. Lappalainen, H. R. Kranzler, R. Malison et al., "A functional neuropeptide Y Leu7Pro polymorphism associated with alcohol dependence in a large population sample from the United States," Archives of General Psychiatry, vol. 59, no. 9, pp. 825831, 2002.

[19] M. D. Koehnke, S. Schick, U. Lutz et al., "Severity of alcohol withdrawal symptoms and the T1128c polymorphism of the neuropeptide Y gene," Journal of Neural Transmission, vol. 109, no. 11, pp. 1423-1429, 2002.

[20] G. Zhu, L. Pollak, S. Mottagui-Tabar et al., "NPY leu7pro and alcohol dependence in Finnish and Swedish populations," Alcoholism: Clinical and Experimental Research, vol. 27, no. 1, pp. 19-24, 2003.

[21] U. Jaakkola, U. Pesonen, E. Vainio-Jylhä, M. Koulu, M. Pöllönen, and J. Kallio, "The Leu7Pro polymorphism of neuropeptide $\mathrm{Y}$ is associated with younger age of onset of type 2 diabetes mellitus and increased risk for nephropathy in subjects with diabetic retinopathy," Experimental and Clinical Endocrinology and Diabetes, vol. 114, no. 4, pp. 147-152, 2006.

[22] C. T. M. Van Rossum, H. Pijl, R. A. H. Adan, B. Hoebee, and J. C. Seidell, "Polymorphisms in the NPY and AGRP genes and body fatness in Dutch adults," International Journal of Obesity, vol. 30, no. 10, pp. 1522-1528, 2006.

[23] M. E. Mangan, J. M. Williams, S. M. Lathe, D. Karolchik, and W. C. Lathe, "UCSC genome browser: deep support for molecular biomedical research," Biotechnology Annual Review, vol. 14, pp. 63-108, 2008.

[24] D. Karolchik, A. S. Hinrichs, and W. J. Kent, "The UCSC genome browser," in Current Protocols in Bioinformatics, chapter 1, 2007. 
[25] N. S. Kim, S. M. Oh, M. M. Ko, M. H. Cha, B. K. Kang, and O. S. Bang, "Association of the C-399T promoter polymorphism of neuropeptide Y with susceptibility to ischemic stroke," Clinical Biochemistry, vol. 42, no. 16-17, pp. 1699-1704, 2009.

[26] J. T. Yu, N. N. Yu, S. S. Gao et al., "Neuropeptide Y polymorphisms and ischemic stroke in Chinese population," Clinica Chimica Acta, vol. 411, no. 3-4, pp. 242-245, 2010.

[27] H. J. Kim, H. S. Bae, S. U. Park, S. K. Moon, J. M. Park, and W. S. Jung, "Clinical approach to the standardization of oriental medical diagnostic pattern identification in stroke patients," Evidence-based Complementary and Alternative Medicine, vol. 2011, Article ID 768492, 7 pages, 2011.

[28] WHO, "WHO international standard terminologies on traditional medicine in the western pacific region," in WHO Western Pacific Regional Office, 2007.

[29] H. Y. Go, Y. K. Kim, B. K. Kang et al., "Report on the Korean standard differentiation of the symptoms and signs for the stroke-2," Korean Journal of Oriental Physiology \& Pathology, vol. 20, pp. 1789-1791, 2006.

[30] I. K. Min, C. H. Kim, J. W. Hwang et al., "The relation of Dampness-Phlegm and metabolic syndrome in acute stroke patients," Journal of Korean Oriental Medicine, vol. 30, no. 1, pp. 109-119, 2009.

[31] J. H. Lim, M. M. Ko, J. S. Lee, O. S. Bang, and M. H. Cha, "Genetic association of SNPs located at PON1 gene with Dampness and phelgm pattern identification among Korea stroke patients," Korean Jourrnal of Oriental Internal Medicine, vol. 31, no. 4, pp. 752-762, 2010.

[32] T. Ouyang, J. N. Song, Y. Miao et al., "Study on relationship between polymorphism of apolipoprotein E gene and syndromes of phlegm and blood stasis in patients with coronary heart disease," Journal of Chinese Integrative Medicine, vol. 3, no. 6, pp. 438-442, 2005.

[33] S. An, E. Li, and X. Tong, "Study on relationship between estrogen receptor gene polymorphism and syndrome differentiation typing of female postmenopausal osteoporosis in Traditional Chinese medicine," Chinese Journal of Integrated Traditional and Western Medicine, vol. 20, no. 12, pp. 907-910, 2000.

[34] S. Chen, F. Lv, J. Gao et al., "HLA class II polymorphisms associated with the physiologic characteristics defined by traditional Chinese medicine: linking modern genetics with an ancient medicine," Journal of Alternative and Complementary Medicine, vol. 13, no. 2, pp. 231-239, 2007.

[35] P. W. Hedrick, "Gametic disequilibrium measures: proceed with caution," Genetics, vol. 117, no. 2, pp. 331-341, 1987.

[36] J. K. Kim, I. C. Seol, I. Lee, H. K. Jo, B. C. Yu, and S. M. Choi, "Report on the Korean standard differentiation of the symptoms and signs for the stroke-1," Korean Journal of Oriental Physiology \& Pathology, vol. 20, pp. 229-234, 2006.

[37] M. K. Karvonen, U. Pesonen, M. Koulu et al., "Association of a leucine(7)-to-proline(7) polymorphism in the signal peptide of neuropeptide $\mathrm{Y}$ with high serum cholesterol and LDL cholesterol levels," Nature Medicine, vol. 4, no. 12, pp. 1434$1437,1998$.

[38] B. Ding, B. Kull, Z. Liu et al., "Human neuropeptide Y signal peptide gain-of-function polymorphism is associated with increased body mass index: possible mode of function," Regulatory Peptides, vol. 127, no. 1-3, pp. 45-53, 2005.

[39] M. Koulu, S. Movafagh, J. Tuohimaa et al., "Neuropeptide $\mathrm{Y}$ and Y2-receptor are involved in development of diabetic retinopathy and retinal neovascularization," Annals of Medicine, vol. 36, no. 3, pp. 232-240, 2004.
[40] B. Ding, "Distribution of the NPY 1128C allele frequency in different populations," Journal of Neural Transmission, vol. 110, no. 11, pp. 1199-1204, 2003.

[41] F. Nyström, P. Nilsson, A. G. Olsson, B. E. Karlberg, and K. P. Öhman, "A population study of plasma neuropeptide Y: correlations with components of the metabolic syndrome," Blood Pressure, vol. 5, no. 6, pp. 349-353, 1996.

[42] M. Itokawa, M. Arai, S. Kato et al., "Association between a novel polymorphism in the promoter region of the neuropeptide Y gene and schizophrenia in humans," Neuroscience Letters, vol. 347, no. 3, pp. 202-204, 2003.

[43] P. R. Buckland, B. Hoogendoorn, C. A. Guy et al., "A high proportion of polymorphisms in the promoters of brain expressed genes influences transcriptional activity," Biochimica et Biophysica Acta-Molecular Basis of Disease, vol. 1690, no. 3, pp. 238-249, 2004. 


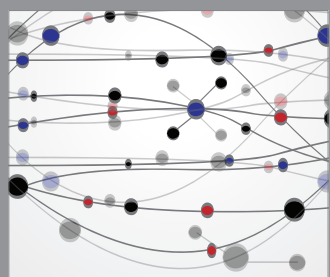

The Scientific World Journal
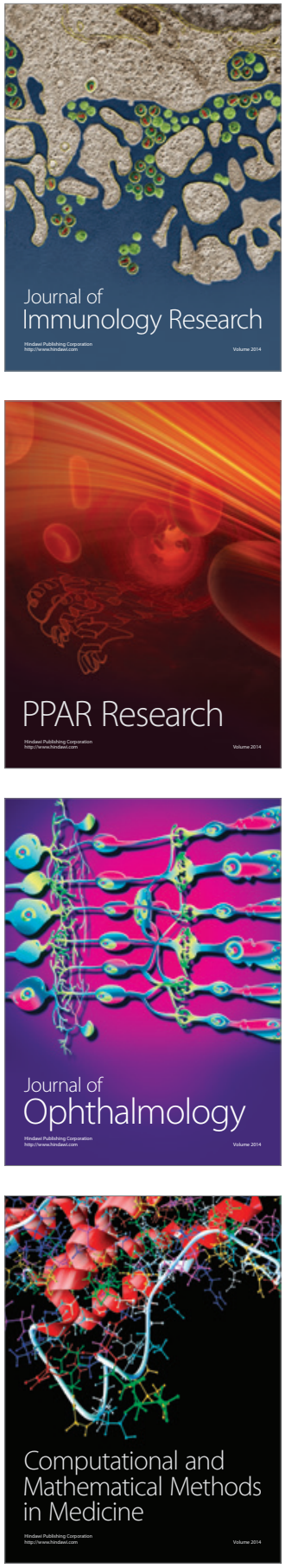

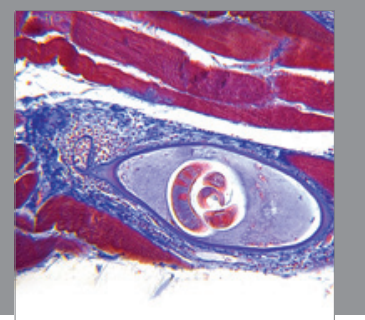

Gastroenterology

Research and Practice
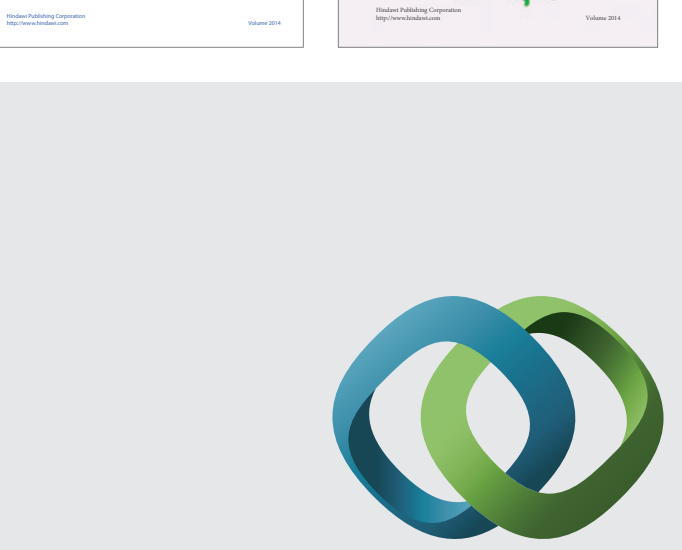

\section{Hindawi}

Submit your manuscripts at

http://www.hindawi.com
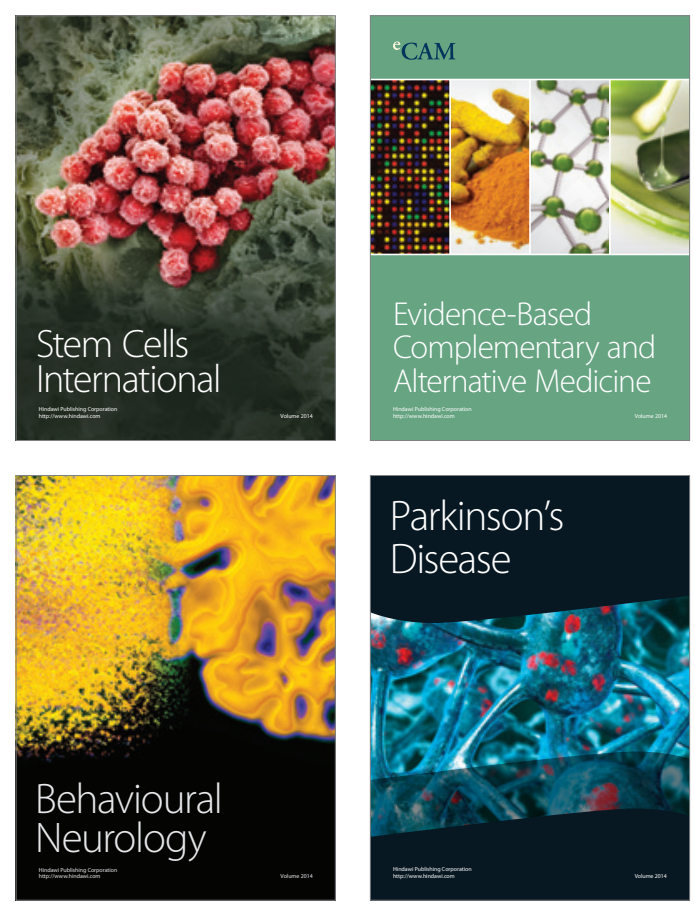

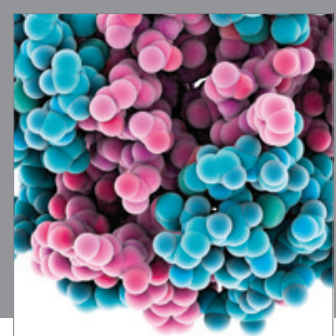

Journal of
Diabetes Research

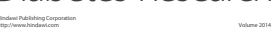

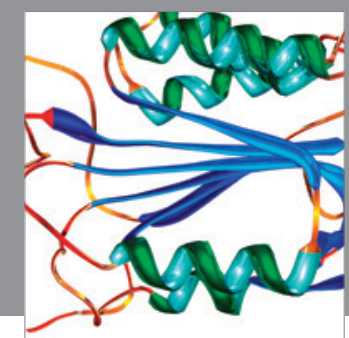

Disease Markers
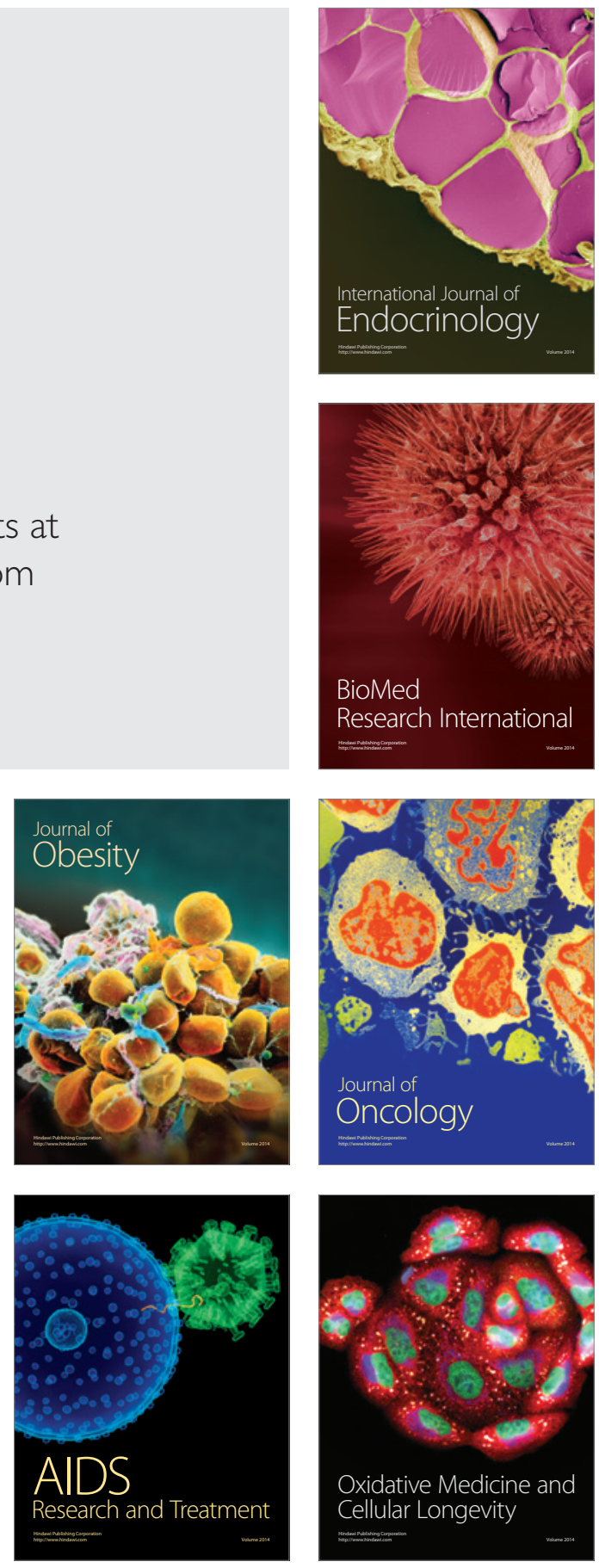